\title{
Shrinking multiplexed orbital angular momentum to the nanoscale
}

\author{
Chao He $\mathbb{1}^{1 凶}$, Yijie Shen $\mathbb{D}^{2}$, Andrew Forbes $\mathbb{B}^{3}$ and Martin J. Booth (1)
}

\begin{abstract}
Orbital angular momentum interactions at the nanoscale have remained elusive because the phase structure becomes unresolved. Now researchers have shown how to overcome this with tightly focused beams, demonstrating a recordhigh six-dimensional encoding in an ultra-dense nanoscale volume.
\end{abstract}

The demand for ultrafast, condensed, broadband and secure transfer of energy and information is advancing at an unprecedented pace, with $6 \mathrm{G}$ telecommunication already on its way. An enabler of this is to exploit light's many degrees of freedom (DoF) for optical multiplexing technology, now an essential tool for modern information delivery. The use of ultrafast pulsed lasers has facilitated the transition of data recording from a surface layer to a $3 \mathrm{D}$ volume $^{1}$. Advances in nanotechnology, especially plasmonic nanomaterials, have enabled light multiplexing to be performed with five DoFs ${ }^{2}$. An exciting prospect to overcome the impending data $\mathrm{crunch}^{3}$ is to use the spatial mode of light as an additional DoF, with orbital angular momentum (OAM) a popular candidate ${ }^{4}$. But modern optical information technology systems require miniaturised and integrated solutions, and here OAM has a problem: as one zooms into the nanoscale, the telltale helical structure of OAM gets lost as the light wave appears locally flat. Just as ants cannot easily tell whether they are walking along a straight rod or a helical corkscrew, nanostructured matter cannot discern the OAM of a spatial mode. In a recently published paper ${ }^{5}$, Xu Ouyang and co-authors overcome this challenge in an ingenious manner. They note that while the OAM phase itself

Correspondence: Chao He (chao.he@eng.ox.ac.uk)

'Department of Engineering Science, University of Oxford, Parks Road, Oxford OX1 3PJ, UK

2Optoelectronics Research Centre, University of Southampton, Southampton SO17 1BJ, UK

Full list of author information is available at the end of the article cannot be detected, its influence on the resulting subwavelength polarisation structure can. In a tightly focussed OAM beam, fine polarisation ellipses arise with orientations dictated by the OAM charge. This orientation can be detected by nanostructures with a similar orientation. Using this as a tool, the authors demonstrate that sixdimensional optical OAM multiplexing can be realised at the nanoscale, exploiting wavelength, polarisation, and three spatial dimensions simultaneously (Fig. 1).

A combination of tight focusing and synthetic helical dichroism (HD) play important roles in this breakthrough. High numerical-aperture (NA) lenses, which are essential for tight beam focusing, feature intrinsic polarisation aberrations $^{6,7}$ (for example, owing to Fresnel and geometric effects). One aspect of its overall representation is that in the nonparaxial vectorial electric field, spatial variations exist in the state of polarisation, which varies for different incident OAM beams. While commonly seen as a nuisance (e.g., for polarisation imaging) or overlooked (e.g., in optical multiplexing), this property is in contrast harnessed by the team in conjunction with the synthetic HD. Dichroism, a material property that induces diattenuation during light propagation, has been used in many research fields, ranging from quantum physics to clinical applications. In principle, it is associated with a difference in absorption coefficients for orthogonal polarisation states, resulting in different polarisation responses that are encoded in the corresponding materials. The team describes and leverages the synthetic HD of a nanostructure under the excitation of OAM beams with respect

\section{(c) The Author(s) 2021}

(c) Open Access This article is licensed under a Creative Commons Attribution 4.0 International License, which permits use, sharing, adaptation, distribution and reproduction in any medium or format, as long as you give appropriate credit to the original author(s) and the source, provide a link to the Creative Commons license, and indicate if changes were made. The images or other third party material in this article are included in the article's Creative Commons license, unless indicated otherwise in a credit line to the material. If material is not included in the article's Creative Commons license and your intended use is not permitted by statutory regulation or exceeds the permitted use, you will need to obtain permission directly from the copyright holder. To view a copy of this license, visit http://creativecommons.org/licenses/by/4.0/. 


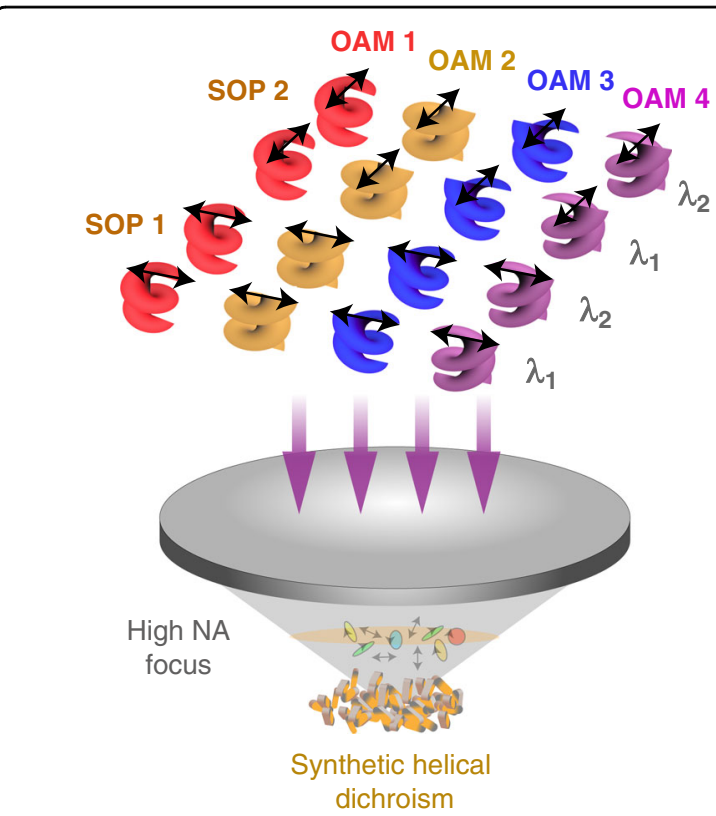

Fig. 1 Illustrations of six-dimensional OAM multiplexing. SOP state of polarisation, $\lambda$ wavelength

to the topological charge, linear polarisation, and wavelength. Such synthetic HD is polarisation-sensitive, hence can be utilised together with the different polarisation ellipses in the tightly focused beam that link with different OAM charges, enabling sensitive OAM multiplexing at the nanoscale. Compared with conventional HD in chiral structures, this synthetic HD is not restricted to opposite topological charges, hence provides more versatile possibilities $^{5,8}$.

The team utilise these properties to demonstrate sixdimensional optical encryption with nanometric $Q R$ codes via OAM division regarding two wavelengths and orthogonal incident polarisation states. Their success also build upon plasmonic coupling effects that introduce hotspots with remarkably augmented local fields to enhance synthetic HD, and two-photon luminescence for an intuitive indication of the synthetic HD. These phenomena are of significant benefit in the encoding and decoding processes. Furthermore, the six-dimensional encoded images are retrieved from different layers of the coupled disordered nanoparticle aggregates. It is worth noting that the sensitivity of the photothermal deformation of gold nanorods and the two-photon luminescence contrast can both be boosted considerably by the enhanced HD, hence benefiting the multiplexing process via improved signals.

The results obtained by the team open exciting possibilities of multiplexing the OAM division for highinformation capacity and security. Building upon these advances in six-dimensional multiplexing, there is still work to be done. The polarisation effects vary between different high NA lenses and these effects can disorder the traceable polarisation ellipses in the focal region. Hence, cross-talk could occur between different OAM channels. However, this is likely to be conquered with engineering solutions through lens optimisation. There are also prospects for further extensions, such as towards higher-dimensional structured light multiplexing. Recent advances in structured light ${ }^{9}$ have pushed the limits of what is possible. The present work highlights the exciting prospects in marrying structured light with structured matter, for control at scales from the large to the small.

\section{Author details}

${ }^{1}$ Department of Engineering Science, University of Oxford, Parks Road, Oxford OX1 3PJ, UK. ²Optoelectronics Research Centre, University of Southampton, Southampton SO17 1BJ, UK. ${ }^{3}$ School of Physics, University of the Witwatersrand, Private Bag 3, Johannesburg 2050, South Africa

\section{Conflict of interest}

The authors declare no competing interests.

Published online: 28 October 2021

References

1. Parthenopoulos, D. A. \& Rentzepis, P. M. Three-dimensional optical storage memory. Science 245, 843-845 (1989).

2. Zijlstra, P., Chon, J. W. M. \& Gu, M. Five-dimensional optical recording mediated by surface plasmons in gold nanorods. Nature 459, 410-413 (2009).

3. Richardson, D. J., Fini, J. M. \& Nelson, L. E. Space-division multiplexing in optical fibres. Nat. Photonics 7, 354-362 (2013).

4. Willner, A. E. et al. Optical communications using orbital angular momentum beams. Adv. Opt. Photonics 7, 66-106 (2015).

5. Ouyang, Xu. et al. Synthetic helical dichroism for six-dimensional optical orbital angular momentum multiplexing. Nat. Photonics https:/doi.org/10.1038/ s41566-021-00880-1 (2021).

6. Shribak, M. I., Inoue, S. \& Oldenbourg, R. Polarization aberrations caused by differential transmission and phase shift in high numerical aperture lenses: theory, measurement, and retification. Optical Eng. 41, 943-954 (2002).

7. Yun, G., Crabtree, K. \& Chipman, R. A. Skew aberration: a form of polarization aberration. Opt. Lett. 36, 4062-4064 (2011).

8. Ni, J. C. et al. Giant helical dichroism of single chiral nanostructures with photonic orbital angular momentum. ACS Nano 15, 2893-2900 (2021).

9. Forbes, A., De Oliveira, M. \& Dennis, M. R. Structured light. Nat. Photonics $\mathbf{1 5}$, 253-262 (2021). 